\title{
Publisher Correction: Stabilizing the mood of tumours
}

\section{Anna Dart}

Correction to: Nature Reviews Cancer https://doi.org/10.1038/s41568-021-00422-5, published online 26 October 2021.

In the Table of Contents summary for the originally published In Brief, the expression of PDL1 was inaccurately described as being decreased. This has been corrected in the HTML version of the In Brief to reflect that the expression of PDL1 was in fact increased.

https://doi.org/10.1038/s41568-021-00424-3 I Published online 12 November 2021

(๑) Springer Nature Limited 2021 